\title{
Dissipative dynamics of a harmonically confined Bose-Einstein condensate
}

\author{
Z. Wu and E. Zaremba \\ Department of Physics, Astronomy and Engineeering Physics, \\ Queen's University, Kingston, Ontario KYL 3N6, Canada.
}

(Dated: October 18, 2018)

\begin{abstract}
We study the dissipation of the centre of mass oscillation of a harmonically confined condensate in the presence of a disorder potential. An extension of the Harmonic Potential Theorem allows one to formulate the dynamics from the point of view of an oscillating disorder potential. This formulation leads to a rigorous result for the damping rate in the limit of weak disorder.

PACS numbers: 03.75.Kk, 67.85.De
\end{abstract}

Trapped Bose gases provide an ideal setting for the study of nonequilibrium phenomena in a many-body system. Some examples include condensate formation following a thermal quench 1, 2], collective excitations as a function of temperature [3] and the relaxation of highly nonequilibrium vortex states [4, 5]. In these, and many other situations, the underlying superfluidity plays an essential role in determining the dynamical behaviour.

In some recent experiments [6-8], the dissipative dynamics of a Bose condensate in the presence of a disorder potential was studied. This perturbing potential is the vehicle by which the collective centre of mass motion of the condensate is dissipated by means of internal excitations. The situation is analogous to the motion of an impurity through a superfluid where it is found that excitations can be produced above a critical velocity [9]. Here it is clear that the relative velocity of the impurity and superfluid is the relevant variable; the motion of a heavy impurity through a stationary superfluid or the flow of a superfluid past a stationary obstacle are physically equivalent.

In this paper we demonstrate that a similar symmetry pertains to a Bose gas trapped in a harmonic potential. Harmonic confinement leads to an equivalence between the motion of the condensate through a disorder potential that is at rest relative to the confining potential, and the harmonic motion of the disorder potential itself relative to the trapping potential and the condensate. We exploit this equivalence to formulate a rigorous theory of the centre of mass motion in the presence of a disorder potential and obtain an estimate of the damping in the limit of weak disorder. Our results are in qualitative agreement with those obtained earlier from an analysis of the one-dimensional Gross-Pitaevskii equation [10].

The Hamiltonian of the system studied experimentally is

$$
\begin{aligned}
\widehat{H} & =\sum_{i=1}^{N}\left[\frac{\hat{p}_{i}^{2}}{2 m}+V_{\text {trap }}\left(\hat{\mathbf{r}}_{i}\right)\right]+\widehat{V}_{\mathrm{int}}+\sum_{i=1}^{N} V_{\mathrm{dis}}\left(\hat{\mathbf{r}}_{i}\right) \\
& \equiv \widehat{H}_{0}+\widehat{V}_{\mathrm{dis}}
\end{aligned}
$$

where $V_{\text {trap }}(\mathbf{r})=\frac{1}{2} m\left(\omega_{\perp}^{2} \rho^{2}+\omega_{z}^{2} z^{2}\right)$ is the trapping potential and $V_{\text {dis }}(\mathbf{r})$ is a disorder potential whose properties we specify later. The interactions between the atoms is contained in $\widehat{V}_{\text {int }}$. To begin, we rephrase the Harmonic Potential Theorem (HPT) 11] in a form which will be of particular utility in the subsequent development. Starting with the many-body state $|\Phi\rangle$, we define the state

$$
|\Psi\rangle=\exp \left\{\frac{i}{\hbar}(\mathbf{p} \cdot \widehat{\mathbf{R}}-\mathbf{x} \cdot \widehat{\mathbf{P}})\right\}|\Phi\rangle \equiv \widehat{T}(\mathbf{x}, \mathbf{p})|\Phi\rangle,
$$

where $\widehat{\mathbf{R}}=\frac{1}{N} \sum_{i=1}^{N} \hat{\mathbf{r}}_{i}$ is the centre of mass coordinate and $\widehat{\mathbf{P}}=\sum_{i=1}^{N} \hat{\mathbf{p}}_{i}$ is the total momentum of the particles. These variables satisfy the commutation relation $\left[\widehat{R}_{\mu}, \widehat{P}_{\nu}\right]=i \hbar \delta_{\mu \nu}$. The unitary operator $\widehat{T}$ shifts the state in position space by $\mathbf{x}$ so that $\left|\Psi\left(\mathbf{r}_{1}, \ldots, \mathbf{r}_{N}\right)\right|^{2}=\mid \Phi\left(\mathbf{r}_{1}-\right.$ $\left.\mathbf{x}, \ldots, \mathbf{r}_{N}-\mathbf{x}\right)\left.\right|^{2}$. At the same time, the state is shifted in momentum space by $\mathbf{p} / N$ so that $\left|\tilde{\Psi}\left(\mathbf{p}_{1}, \ldots, \mathbf{p}_{N}\right)\right|^{2}=$ $\left|\tilde{\Phi}\left(\mathbf{p}_{1}-\mathbf{p} / N, \ldots, \mathbf{p}_{N}-\mathbf{p} / N\right)\right|^{2}$. This implies that the total momentum is boosted by $\mathbf{p}$.

We now consider the time evolution of the state $|\Psi\rangle$ according to the unperturbed Hamiltonian $\widehat{H}_{0}$. Using the Heisenberg equations of motion for the operators $\widehat{\mathbf{R}}$ and $\widehat{\mathbf{P}}$ with respect to $\widehat{H}_{0}$, we find that

$$
|\Psi(t)\rangle=e^{-i \widehat{H}_{0} t / \hbar}|\Psi\rangle=\widehat{T}(\mathbf{x}(t), \mathbf{p}(t)) e^{-i \widehat{H}_{0} t / \hbar}|\Phi\rangle,
$$

with

$$
\begin{aligned}
& x_{\mu}(t)=x_{\mu} \cos \omega_{\mu} t+\frac{p_{\mu}}{M \omega_{\mu}} \sin \omega_{\mu} t \\
& p_{\mu}(t)=p_{\mu} \cos \omega_{\mu} t-M \omega_{\mu} x_{\mu} \sin \omega_{\mu} t,
\end{aligned}
$$

where $M=m N$ is the total mass of the system. If we now take $|\Phi\rangle$ to be an eigenstate $\left|\Phi_{\alpha}\right\rangle$ of $\widehat{H}_{0}$, we see that $\left|\Psi\left(\mathbf{r}_{1}, \ldots, \mathbf{r}_{N}, t\right)\right|^{2}=\left|\Phi_{\alpha}\left(\mathbf{r}_{1}-\mathbf{x}(t), \ldots, \mathbf{r}_{N}-\mathbf{x}(t)\right)\right|^{2}$. In other words, the probability density rigidly follows the motion of the centre of mass of the system. This is essentially the content of the HPT. More generally, the system can be described by the density matrix $\hat{\rho}=$ $\sum_{\alpha} p_{\alpha}\left|\Phi_{\alpha}\right\rangle\left\langle\Phi_{\alpha}\right|$. If the states $\left|\Phi_{\alpha}\right\rangle$ are all eigenstates of $\widehat{H}_{0}$, the total density of the system for the density matrix $\widehat{T}(\mathbf{x}, \mathbf{p}) \hat{\rho} \widehat{T}^{\dagger}(\mathbf{x}, \mathbf{p})$ oscillates rigidly according to $n(\mathbf{r}, t)=$ $n_{0}(\mathbf{r}-\mathbf{x}(t))$ where $n_{0}(\mathbf{r})=\operatorname{Tr}(\hat{\rho} \hat{n}(\mathbf{r}))$. This applies to the special case of a thermal equilibrium distribution.

We next consider the dynamics of the centre of mass as governed by the full Hamiltonian including the disorder 
potential. The Heisenberg equations of motion lead to the equation

$$
\frac{d^{2} Z}{d t^{2}}+\omega_{z}^{2} Z=\frac{F}{M}
$$

where $Z(t)=\left\langle\Psi(t)\left|\widehat{R}_{z}\right| \Psi(t)\right\rangle$ and $F(t)=\left\langle\Psi(t)\left|\widehat{F}_{z}\right| \Psi(t)\right\rangle$ with $\widehat{F}_{z}=-\sum_{i=1}^{N} \partial V_{\text {dis }}\left(\hat{\mathbf{r}}_{i}\right) / \partial \hat{z}_{i}$. Eq. (6) is an exact statement of the centre of mass dynamics, but requires knowledge of the dynamical state $|\Psi(t)\rangle$. To determine this state we go to the interaction picture and define $\left|\Psi_{I}(t)\right\rangle \equiv \exp \left(i \widehat{H}_{0} t / \hbar\right)|\Psi(t)\rangle$ which satisfies

$$
\left|\Psi_{I}(t)\right\rangle=|\Psi(0)\rangle-\frac{i}{\hbar} \int_{0}^{t} d t^{\prime} \hat{V}_{\mathrm{dis}, I}\left(t^{\prime}\right)\left|\Psi_{I}\left(t^{\prime}\right)\right\rangle
$$

with $\widehat{V}_{\text {dis }, I}(t)=\exp \left(i \widehat{H}_{0} t / \hbar\right) \widehat{V}_{\text {dis }} \exp \left(-i \widehat{H}_{0} t / \hbar\right)$.

In the experiment we consider [7], the centre of mass motion of the condensate is initiated by a sudden shift of the confining harmonic potential in the $z$-direction. To describe this situation, we define the Hamiltonian of the system for $t \leq 0$ to be

$$
\widehat{H}^{\prime}=\sum_{i=1}^{N}\left[\frac{\hat{p}_{i}^{2}}{2 m}+V_{\mathrm{trap}}\left(\hat{\mathbf{r}}_{i}-\mathbf{x}\right)\right]+\widehat{V}_{\mathrm{int}}+\sum_{i=1}^{N} V_{\mathrm{dis}}\left(\hat{\mathbf{r}}_{i}\right)
$$

while for $t>0$, the system evolves according to the Hamiltonian (11). The trap potential in (8) is illustrated by the dashed curve in Fig. 1(a); we assume that the state of the system at $t=0$ is $|\Psi(0)\rangle=\left|\Psi_{0}\right\rangle$, the ground state of $\widehat{H}^{\prime}$. This Hamiltonian can be expressed as $\widehat{H}^{\prime}=\widehat{T}(\mathbf{x}, \mathbf{p}) \widetilde{H} \widehat{T}^{\dagger}(\mathbf{x}, \mathbf{p})$, where $\mathbf{x}=z_{0} \hat{\mathbf{z}}, \mathbf{p}=0$ and $\widetilde{H}$ is

$$
\widetilde{H}=\sum_{i=1}^{N}\left[\frac{\hat{p}_{i}^{2}}{2 m}+V_{\text {trap }}\left(\hat{\mathbf{r}}_{i}\right)\right]+\widehat{V}_{\mathrm{int}}+\sum_{i=1}^{N} V_{\mathrm{dis}}\left(\hat{\mathbf{r}}_{i}+\mathbf{x}\right) .
$$

The external potentials of this Hamiltonian are illustrated in Fig. 1(b). The state $\left|\widetilde{\Psi}_{0}\right\rangle=\widehat{T}^{\dagger}(\mathbf{x}, \mathbf{p})\left|\Psi_{0}\right\rangle$ is the ground state of $\widetilde{H}$.

Using the assumed initial state in (7), we find that the state $\left|\widetilde{\Psi}_{I}(t)\right\rangle=\widehat{T}^{\dagger}(\mathbf{x}, \mathbf{p})\left|\Psi_{I}(t)\right\rangle$ satisfies the equation

$$
\left|\widetilde{\Psi}_{I}(t)\right\rangle=\left|\widetilde{\Psi}_{0}\right\rangle-\frac{i}{\hbar} \int_{0}^{t} d t^{\prime} \widetilde{V}_{\mathrm{dis}, I}\left(\mathbf{x}\left(t^{\prime}\right), t^{\prime}\right)\left|\widetilde{\Psi}_{I}\left(t^{\prime}\right)\right\rangle .
$$

where

$$
\begin{aligned}
\widetilde{V}_{\mathrm{dis}, I}(\mathbf{x}(t), t) \\
\quad \equiv \widehat{T}^{\dagger}(\mathbf{x}, \mathbf{p}) \widehat{V}_{\mathrm{dis}, I}(t) \widehat{T}(\mathbf{x}, \mathbf{p}) \\
=e^{i \widehat{H}_{0} t / \hbar} \widehat{T}^{\dagger}(\mathbf{x}(t), \mathbf{p}(t)) \widehat{V}_{\mathrm{dis}} \widehat{T}(\mathbf{x}(t), \mathbf{p}(t)) e^{-i \widehat{H}_{0} t / \hbar} \\
=e^{i \widehat{H}_{0} t / \hbar} \sum_{i=1}^{N} V_{\operatorname{dis}}\left(\widehat{\mathbf{r}}_{i}+\mathbf{x}(t)\right) e^{-i \widehat{H}_{0} t / \hbar}
\end{aligned}
$$

We thus see that $\left|\widetilde{\Psi}_{I}(t)\right\rangle$ is the state that evolves from $\left|\widetilde{\Psi}_{0}\right\rangle$ as a result of an oscillating disorder potential. (a)
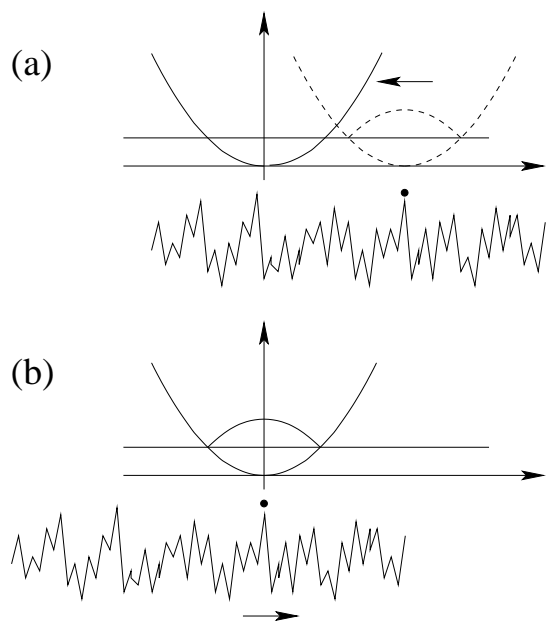

FIG. 1: (a) The condensate, originally in equilibrium with the unshifted trap (dashed) and the disorder potential, begins to oscillate about the centre of the shifted trap (solid). (b) The condensate, originally in equilibrium with the trap and disorder potential, is driven by an oscillating disorder potential.

These results imply that the force appearing in (6) can be expressed as

$$
F(t)=\left\langle\Psi(t)\left|\widehat{F}_{z}\right| \Psi(t)\right\rangle=\left\langle\widetilde{\Psi}_{I}(t)\left|\widetilde{F}_{z, I}(t)\right| \widetilde{\Psi}_{I}(t)\right\rangle,
$$

where

$$
\widetilde{F}_{z, I}(t)=-e^{i \widehat{H}_{0} t / \hbar} \sum_{i=1}^{N} \frac{\partial V_{\operatorname{dis}}\left(\hat{\mathbf{r}}_{i}+\mathbf{x}(t)\right)}{\partial \hat{z}_{i}} e^{-i \widehat{H}_{0} t / \hbar}
$$

Eq. (12) is a key result and shows that there is an intimate connection between the two very distinct physical situations depicted in Fig. 1 In the first, one starts with an excited state corresponding to a displaced condensate. This state then evolves according to (7) in the presence of a static disorder potential. Even though the condensate follows a damped trajectory that eventually ends with the cloud being in equilibrium with the static disorder, the total energy of the system is conserved during this evolution. In the alternative situation described by (10), the condensate starts in its ground state and is driven by a dynamic disorder potential moving according to the unperturbed centre of mass motion. In this case, the dynamic perturbation continually excites the condensate and the total energy increases as a function of time. However, the atomic cloud eventually reaches a steady state in which it moves with the disorder potential with no further increase in energy. That the cloud experiences the same force due to the disorder in these two situations is by no means obvious and is a consequence of the fact that the system is harmonically confined.

More quantitatively, the solution of (6) for the assumed initial conditions is

$$
Z(t)=z_{0} \cos \omega_{z} t+\frac{1}{M \omega_{z}} \int_{0}^{t} d t^{\prime} \sin \omega_{z}\left(t-t^{\prime}\right) F\left(t^{\prime}\right),
$$


where, because of (12), the second term on the right hand side is in fact the displacement for the state $\left|\widetilde{\Psi}_{I}(t)\right\rangle$. From (14) we see that the change in the centre of mass position over one period $T=2 \pi / \omega_{z}$ is

$$
\Delta Z_{l} \equiv Z\left(T_{l}\right)-Z\left(T_{l-1}\right)=-\frac{1}{M \omega_{z}} \int_{T_{l-1}}^{T_{l}} d t \sin \omega_{z} t F(t),
$$

where $T_{l} \equiv l T$. This is valid for both the static and dynamic disorder potential scenarios.

To analyze the effects of $F(t)$ on the dynamics of the centre of mass motion we will assume that the damping it gives rise to is weak. In keeping with this assumption, we evaluate $F(t)$ perturbatively. To second order in the disorder potential we have

$$
\begin{aligned}
F(t)= & \left\langle\Phi_{0}\left|\widetilde{F}_{z, I}(t)\right| \Phi_{0}\right\rangle \\
& -\frac{i}{\hbar} \int_{-\infty}^{0} d t^{\prime} e^{\eta t^{\prime}}\left\langle\Phi_{0}\left|\left[\widetilde{F}_{z, I}(t), \widetilde{V}_{\mathrm{dis}, I}\left(\mathbf{x}, t^{\prime}\right)\right]\right| \Phi_{0}\right\rangle \\
& -\frac{i}{\hbar} \int_{0}^{t} d t^{\prime}\left\langle\Phi_{0}\left|\left[\widetilde{F}_{z, I}(t), \widetilde{V}_{\mathrm{dis}, I}\left(\mathbf{x}\left(t^{\prime}\right), t^{\prime}\right)\right]\right| \Phi_{0}\right\rangle,
\end{aligned}
$$

where $\left|\Phi_{0}\right\rangle$ is the ground state of $\widehat{H}_{0}$. The second term on the right hand side of (16) involving the positive infinitesimal $\eta$ accounts for the lowest order effect of the disorder on the ground state $\left|\widetilde{\Psi}_{0}\right\rangle$, while the third term arises from the dynamic perturbation of $\left|\widetilde{\Psi}_{I}(t)\right\rangle$ in (10).

We now write

$$
\widetilde{V}_{\mathrm{dis}, I}(\mathbf{x}(t), t)=\int d \mathbf{r} V_{\mathrm{dis}}(\mathbf{r}+\mathbf{x}(t)) \hat{n}_{I}(\mathbf{r}, t),
$$

where $\hat{n}_{I}(\mathbf{r}, t)$ is the density operator in the interaction picture. The disorder potential is represented as

$$
V_{\text {dis }}(\mathbf{r})=\int \frac{d \mathbf{k}}{(2 \pi)^{3}} e^{i \mathbf{k} \cdot \mathbf{r}} U(\mathbf{k}),
$$

where the Fourier amplitudes $U(\mathbf{k})$ are stochastic variables having the following disorder averages:

$$
\overline{U(\mathbf{k})}=0, \quad \overline{U(\mathbf{k}) U^{*}\left(\mathbf{k}^{\prime}\right)}=(2 \pi)^{3} \delta\left(\mathbf{k}-\mathbf{k}^{\prime}\right) R(\mathbf{k}) .
$$

Inserting (17) into (16) and performing the disorder average, we find that $\overline{\overline{F(t)}}=\overline{F_{1}(t)}+\overline{F_{2}(t)}$ with

$$
\begin{aligned}
& \overline{F_{1}(t)}=i \int_{-\infty}^{0} d t^{\prime} e^{\eta t^{\prime}} \int \frac{d \mathbf{k}}{(2 \pi)^{3}} R(\mathbf{k}) k_{z} e^{i \mathbf{k} \cdot[\mathbf{x}(t)-\mathbf{x}]} \chi\left(\mathbf{k}, \mathbf{k} ; t-t^{\prime}\right), \\
& \overline{F_{2}(t)}=i \int_{0}^{t} d t^{\prime} \int \frac{d \mathbf{k}}{(2 \pi)^{3}} R(\mathbf{k}) k_{z} e^{i \mathbf{k} \cdot\left[\mathbf{x}(t)-\mathbf{x}\left(t^{\prime}\right)\right]} \chi\left(\mathbf{k}, \mathbf{k} ; t-t^{\prime}\right),
\end{aligned}
$$

where $\chi\left(\mathbf{k}, \mathbf{k} ; t-t^{\prime}\right)$ is the Fourier transform of the density response function

$$
\chi\left(\mathbf{r}, \mathbf{r}^{\prime} ; t-t^{\prime}\right)=\frac{i}{\hbar} \theta\left(t-t^{\prime}\right)\left\langle\Phi_{0}\left|\left[\hat{n}_{I}(\mathbf{r}, t), \hat{n}_{I}\left(\mathbf{r}^{\prime}, t^{\prime}\right)\right]\right| \Phi_{0}\right\rangle .
$$

The disorder averaged force in (20) is the main result of this paper and will be used to estimate the damping of the centre of mass motion in the linear response regime.

In the experiments [7], the speckle pattern is onedimensional so that $R(\mathbf{k})=(2 \pi)^{2} \delta\left(k_{x}\right) \delta\left(k_{y}\right) R\left(k_{z}\right)$ with

$$
R\left(k_{z}\right)=\sqrt{\pi} \sigma \overline{V_{\mathrm{dis}}^{2}} e^{-\frac{1}{4} \sigma^{2} k_{z}^{2}},
$$

where $\sigma$ is the correlation length of the gaussian disorder and $\overline{V_{\text {dis }}^{2}}$ is the square of the standard deviation of the disorder potential. In this situation, the response function of interest is

$$
\chi\left(k_{z}, k_{z} ; \tau\right)=\int d^{3} r \int d^{3} r^{\prime} e^{i k_{z}\left(z-z^{\prime}\right)} \chi\left(\mathbf{r}, \mathbf{r}^{\prime} ; \tau\right) .
$$

A formal expression for this quantity can be given in terms of the exact Bogoliubov excitations of the system. However, here we make use of a local density approximation (LDA) whereby each element along the length of the condensate is treated as part of a uniform cylindrical condensate having a density per unit length of $\nu(z)$. In this approximation, the response function is taken to be

$$
\chi(k, k ; \tau) \simeq \int d z \chi_{\mathrm{cyl}}(k, \tau ; \nu(z)),
$$

where $\chi_{\text {cyl }}(k, \tau ; \nu(z))$ is the density response function of a uniform cylindrical condensate. Here and in the following we drop the $z$ subscript on $k_{z}$ for convenience. In the Bogoliubov approximation,

$\chi_{\mathrm{cyl}}(k, \tau ; \nu(z))=\frac{i}{\hbar} \theta(\tau) \sum_{j} \psi_{j}^{2}(k)\left(e^{-i \omega_{j}(k) \tau}-e^{i \omega_{j}(k) \tau}\right)$,

where $\psi_{j}(k)=2 \pi \int_{0}^{\infty} d \rho \rho \delta n_{j}(\rho, k)$ is the cross-sectional average of the mode density fluctuation $\delta n_{j}(\rho, k)$; the index $j$ distinguishes the various radial modes of the cylindrical condensate.

We calculate the density fluctuation by treating the condensate in the Thomas-Fermi (TF) approximation [12]. This is a good approximation in the experimental context since the number of atoms in the cloud is of order $10^{6}$. In the hydrodynamic limit, the normalization of the density fluctuation is then given by $2 \pi \int_{0}^{R_{\perp}} d \rho \rho \delta n_{j}^{2}(\rho, k)=\hbar \omega_{j}(k) / 2 g$, where $R_{\perp}(z)=$ $\lambda \sqrt{R_{z}^{2}-z^{2}}$ is the transverse TF radius at the position $z$ along the axis. Here, $\lambda=\omega_{z} / \omega_{\perp}, R_{z}=\sqrt{2 \mu / m \omega_{z}^{2}}, \mu$ is the chemical potential and $g=4 \pi a \hbar^{2} / \mathrm{m}$.

It can be shown that the contribution of $\overline{F_{1}(t)}$ is negligible in comparison to $\overline{F_{2}(t)}$. We thus focus on the latter in the following. Substituting (24) together with (25) into (20) we obtain

$$
\begin{aligned}
\overline{F_{2}(t)}= & -\frac{1}{\hbar} \int_{-R_{z}}^{R_{z}} d z \int \frac{d k}{2 \pi} k R(k) \sum_{j} \psi_{j}^{2}(k) \\
& \times \int_{0}^{t} d t^{\prime} e^{i k\left(z(t)-z\left(t^{\prime}\right)\right)}\left(e^{-i \omega_{j}(k)\left(t-t^{\prime}\right)}-\text { c.c }\right) .
\end{aligned}
$$




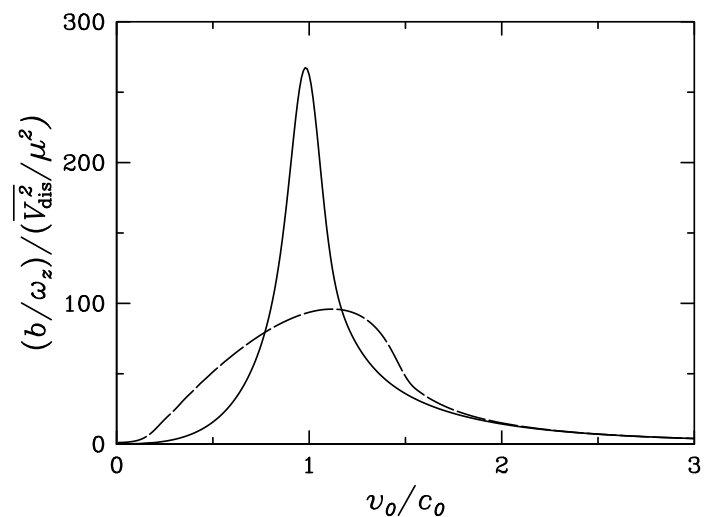

FIG. 2: The damping rate vs. $v_{0} / c_{0}$. The parameters used in this calculation are [7]: $\omega_{z} / 2 \pi=3.6 \mathrm{~Hz} ; \omega_{\perp} / 2 \pi=180$ $\mathrm{Hz}$; the $s$-wave scattering length $a=200 a_{0} ; \mu / h=1 \mathrm{kHz}$; $\sigma=10.6 \mu \mathrm{m}$. The solid curve is for the cylindrical LDA while the dashed curve is for the bulk LDA.

For the initial conditions being considered, $z(t)=$ $z_{0} \cos \omega_{z} t$. In this case, we have

$$
e^{i k z(t)}=\sum_{n=-\infty}^{\infty} e^{i n \pi / 2} J_{n}\left(z_{0} k\right) e^{i \omega_{n} t}
$$

where $\omega_{n}=n \omega_{z}$ and $J_{n}(x)$ is the Bessel function of the first kind of integral order $n$. This Fourier expansion is substituted into (26) and the resulting expression for $\overline{F_{2}(t)}$ is used in (15) to evaluate $\Delta Z_{l}$ explicitly. Remarkably, we find that $\Delta Z_{l}$ is virtually independent of $l$; the $l=1$ result differs from the $l \rightarrow \infty$ limit by a few percent. There is essentially no transient on the time scale of $T$ and implies that $Z\left(T_{l}\right) \simeq z_{0}(1-b l T)$. Defining the damping of the oscillation as $b T=-\Delta Z_{\infty} / z_{0}$, we thus find

$$
\begin{aligned}
\frac{b}{\omega_{z}}=\frac{2 \pi}{M v_{0}^{2} \hbar} \int_{-R_{z}}^{R_{z}} d z \int \frac{d k}{2 \pi} R(k) \sum_{j} \psi_{j}^{2}(k) \\
\quad \times \sum_{n=1}^{\infty} n J_{n}^{2}\left(z_{0} k\right) \delta\left(\omega_{j}(k)-n \omega_{z}\right),
\end{aligned}
$$

where $v_{0}=\omega_{z} z_{0}$.

The results of our calculation of the damping rate based on (28) are shown in Fig. 2. We observe that the damping rate exhibits a resonant peak at $v_{0} / c_{0} \simeq 1$, where $c_{0}=\sqrt{\mu / 2 m}$ is the sound speed in a cylindrical condensate with chemical potential $\mu$ [12]. Also shown in the figure is the result obtained using the bulk LDA [13] whereby each element of the condensate is treated as a homogeneous gas. The cylindrical LDA is an improved approximation since it explicitly accounts for the effect of the transverse confinement on the excitations of the system and becomes exact in the limit of a small aspect ratio $\lambda$ ( $\omega_{z} \rightarrow 0$ with $\mu$ held fixed).
We now compare our results to the measurements presented in Fig. 3 of Ref. 7]. The initial displacement of the harmonic potential of $\sim 700 \mu \mathrm{m}$ corresponds to $v_{0} / c_{0} \simeq 2.9$. For the weakest disorder strength given of $\overline{V_{\mathrm{dis}}^{2}} / \mu^{2}=0.0064$ we find $\left(b / \omega_{z}\right)_{\mathrm{th}} \simeq 0.03$, whereas $\left(b / \omega_{z}\right)_{\exp } \simeq 0.04$ [7]. This should be taken as reasonably good agreement given that there are no adjustable parameters in the calculation. In this regard, we emphasize that the damping rate cannot be adequately characterized using a white-noise spectrum [14].

We have also analyzed the data of Ref. [8] which is obtained using a different protocol to excite the centre of mass oscillation. Here, the disorder is switched on suddenly only after the oscillation of the condensate has been initiated. We find that the linear response damping rate is still given by (28) in this case. Using the experimental parameters corresponding to Fig. 7 of Ref. [8], we obtain $\left(b / \omega_{z}\right)_{\text {th }} \simeq 0.06$ for $v_{0} / c_{0}=2.9$ and $\overline{V_{\text {dis }}^{2}} / \mu^{2}=0.0064$, whereas $\left(b / \omega_{z}\right)_{\exp } \simeq 0.002$ [8]. We have no explanation for this discrepancy. Perhaps the analysis of a different situation such as a gaussian perturbation [8] may shed light on the limitations of the linear response calculation.

In summary, we have shown that the dissipative dynamics of the centre of mass motion can be formulated in terms of a conventional response function approach even though the initial state of the system is far out of equilibrium. With the appropriate response functions, this approach can also be used to study the dissipation at finite temperatures and in fermionic systems.

This work was supported by a grant from NSERC of Canada. We would like to acknowledge useful discussions with Randy Hulet.

[1] H.-J. Miesner et al., Science 279, 1005 (1998).

[2] M. J. Bijlsma, E. Zaremba and H. T. C. Stoof, Phys. Rev. A 62, 063609 (2000).

[3] A. Griffin, T. Nikuni and E. Zaremba, Bose-Condensed Gases at Fintite Temperature (Cambridge, Cambridge, 2009).

[4] J. R. Abo-Shaeer, C. Raman, and W. Ketterle, Phys. Rev. Lett. 88, 070409 (2002).

[5] B. Jackson, N. P. Proukakis, C. F. Barenghi and E. Zaremba, Phys. Rev. A 79, 053615 (2009).

[6] J. E. Lye et al., Phys. Rev. Lett. 95, 070401 (2005).

[7] Y. P. Chen et al, Phys. Rev. A 77, 033632 (2008).

[8] D. Dries, S. E. Pollack, J. M. Hitchcock, and R. G. Hulet, Phys. Rev. A 82, 033603 (2010).

[9] G. E. Astrakharchik and L. P. Pitaevskii, Phys. Rev. A 70, 013608 (2004).

[10] M. Albert, T. Paul, N. Pavloff and P. Leboeuf, Phys. Rev. Lett. 100250405 (2008); Phys. Rev. A 82 011602(R) (2010).

[11] J. F. Dobson, Phys. Rev. Lett. 73, 2244 (1994).

[12] E. Zaremba, Phys. Rev. A 57, 518 (1998).

[13] A. Brunello, F. Dalfovo, L. Pitaevskii, S. Stringari and 
F. Zambelli, Phys. Rev. A 64, 063614 (2001).

[14] S. G. Bhongale, P. Kakashvili, C. J. Bolech and H. Pu,

Phys. Rev. A 82, 053632 (2010). 